and animal souls: we were an encyclopædia for poets searching for spectroscopic metaphors; a hospital for ideas battered by the shocks of criticism ; a home for the consolation of lost causes and the triumph of successful ones: people of all trades, professioñs, races, nationalities, sexes, ages, religionsgreat men, small men, lean men, brawny men, brown men, black men, grey men, tawny men, grave old plodders, gay young friskers-all came for advice to this narrow specialist, and went away, if not always satisfied, at least convinced that nothing further could be done along that line.

All this is now ended, but we have one consolation, and a great one. When Persephone was taken by Pluto from the fields of Enna into the shades below, she was permitted to return once a year for the comfort of those who were left behind. Prof. Fowler is not going into the shades : he is leaving the visible spectrum, but there is a whole octave of ultra-violet to be traversed before the vacuum region is reached. And whatever god presides over the land in which he chooses to dwell, he goes on the condition that, not merely once a year, but whenever instinct tells him that he is needed, he is to be allowed to revisit the glimpses of the arc and the spark and the vacuum tube; and I for one shall listen daily for the familiar footstep. When it comes a drawer will be opened, and out will come the problems and the difficulties that have been put aside to await the coming of the master.

\title{
Fluorescence Microscopy and its Application to the Identification of Fibres
}

$\mathrm{O}^{\mathrm{m}}$ $\mathrm{NE}$ of the most recent and interesting applications of ultra-violet light as a testing method is its use as an aid to microscopical work. Since it is well known that structures visible to the naked eye show distinguishing features in ultra-violet light which are invisible in ordinary light, it is not unnatural that the application to microscopic structures of the same principle should have been attempted. As already indicated ${ }^{2}$, this method has met with considerable success, notably in the examination of sections of botanical specimens such as seeds, tissues, etc., the best results being obtained in cases where the individual details of the structure fluoresce differently. Starch and fatty matters, for example, fluoresce vividly and stand out in sharp contrast, and a notable case is that of cacao, in which the shell tissues and mucilage cells may be distinguished in this way.

The method has now been carried a stage further by the use of fluorescent materials as stains, and this has opened up many promising lines of advance in microscopical technique. It is obvious that if the dyes used for selective staining in ordinary microscopical work are supplemented by substances which cause a particular detail of the structure to fluoresce with a specific colour in ultra-violet light, then many strings will be added to the bow of the practical microscopist. Fortunately a large number of such substances (usually dyes) exist, and only await investigation.

The apparatus required for this work is by no means elaborate. A good microscope and a source of ultra-violet light are of course essential, and in connexion with the latter there seem to be differences of opinion among various workers as to which type gives the best results. All would probably agree, however, that a brilliant point source is desirable, so that the mercury vapour lamp in the form most familiar to workers in Great Britain is by no means the ideal. Successful results have, however, been obtained with arcs struck between iron, cadmium, magnesium-carbon and nickel-carbon electrodes.

The lens system on the arc side should be made of quartz on account of the absorbing powers of ordinary glass for ultra-violet light. This applies to the converging lens and condenser used to concentrate the light on the microscope mirror, and in addition it is desirable to insert filters to remove visible and infrared rays, so that the radiation finally obtained covers the range $3,000-4,000 \mathrm{~m} \mu$. Numerous commercial filters are available for the former purpose, and for the latter a cell containing a solution of copper sulphate is very convenient. Since the microscope is used only for the observation of effects produced by visible light, ordinary glass lenses may be used, and if the fluorescence is sufficiently bright, these are satisfactory even for colour photography.

Useful adjuncts in this work are devices for darkground and surface illumination. The Lieberkuhn mirror and other lens systems used for the latter purpose give very striking results which are quite different from those obtained by transmitted light; the self-luminosity of fluorescent structures also enables vivid effects to be obtained with dark-ground illumination. In some cases, it is an advantage to bed the specimen in a mounting medium, and the selection of the medium should be then governed by the nature of the specimen. Thus both Canada balsam and paraffin wax themselves fluoresce, but whilst in some cases the emission of light masks the fluorescence of the structure, in others it may be used to throw dark structures into relief. A speck of quinine placed on the slide is a great aid in focusing, as it is highly fluorescent.

Even under the above conditions, a certain amount of ultra-violet light may still reach the eye, and it is therefore advisable for prolonged work to wear protecting goggles in order to avoid the possibility of conjunctivitis. Special cover-slips which are opaque to ultra-violet light are an additional precaution and serve the further purpose of eliminating any fluorescence produced by the action of the rays on the lens system. For work with transmitted light, a quartz prism or a silver reflector is used in place of the microscope mirror, and the slide must, of course, be made of quartz; glycerol should replace cedar wood oil for 'oil' immersion lenses.

Some of the most interesting advances in this work have been made in connexion with fibre analysis, and in particular with that branch of the subject which is the concern of the cellulose industries. A number of ordinary stains are already in continual use in this connexion, but they suffer from several deferts, notably that they are difficult to prepare in a sensitive form, and that their applications are limited. F. Noss and H. Sadler ${ }^{2}$, however, have examined the suitability of a large number of dyes from the point of view of fluorescence microscopy, and find that in particular, a 0.05 per cent solution of rhodamine -6 G-D has useful properties. Thus, fibres prepared by the sulphite process appear yellow, whilst' sulphate pulps give an orange-red colour; if the fibres have not been bleached the colours assume a brown tinge. Since the vivid blue fluorescence of unstained, unbleached wood fibres is a well-known phenomenon, 
it is therefore possible first to determine the amount of this material present and then, by staining, to ascertain by what process it has been prepared.

This work has more recently been taken up and extended in a series of publications by B. Schulze and E. Göthel ${ }^{3}$. They also examined a large number of dyes and confirm the suitability of rhodamine and eosin, and in addition, recommend sulphorhodamine$G$ and certain of the flavophosphines; the former may be used to distinguish unbleached soda pulp from bleached soda and sulphite pulps, in the absence of unbleached sulphite pulp. Brilliant dianil green was also found to be a useful aid to the distinction of summer and spring woods, with which it gives a blue and yellow colour, respectively. Adansonia (light blue) could be differentiated from manilla and jute, and diagnostic details of other unusual fibres such as Gampi, Mitsumata and Kodzu were also obtained; in some cases these methods have been developed quantitatively. A word of caution seems desirable in connexion with the use of rhodamine6- $G$ for fluorescence experiments, as the writer has found that dyes from different sources sold under this name may give entirely different effects; this indeed may explain the failure of Schulze and Gothel to confirm some of the results of Noss and Sadler where this dye has been involved.

Reference should also be made to the work of $\mathbf{E}$. Grunsteidl ${ }^{4}$ in which similar principles are applied to textile fibres. The primary fluorescence of such fibres, that is, the fluorescence obtained in the unstained state, is itself of value for diagnostic purposes, raw cotton being bright blue (dull grey if mercerised), whilst various silks (real and 'artificial') may be differentiated if examined under controlled conditions. The use of dyes and stains, however, and in particular of quinosol followed by alkali, has proved an additional aid; for example, linen then appears yellow and cotton violet. Similarly $\mathbf{A}$. Segitz ${ }^{5}$ records the use of extract of spruce bark for the differentiation of materials of the "Cellophane" type.

These examples are necessarily restricted in use and as yet incompletely investigated, but they at least serve to illustrate the possibilities awaiting investigation in this field.

J. G.

\section{NATURE, 133, 124, Jan. 27, 1934.}

2 Pap. Fabrikant, 31, 413 ; 1933. Korn, ibid., 32, 181; 1934

${ }^{3}$ ibid., 32, 110; 1934. Zellstoff Pap., 14, 93; 1934. Woch. Pap.

No. $7 ; 1934$

Faserforschung, 10, 215 ; 1933. Rayon and Melliand Text. Month.

, 88,$93 ; 1934$

Pap. Fabrikant, 28, 206; 1930.

\section{Microplankton and Hydrography of the Great Barrier Reef*}

$\mathrm{M}^{1}$ ISS S. M. MARSHALL, in her contribution to the Scientific Reports of the Great Barrier Reef Expedition, describes the production of microplankton in the Great Barrier Reef throughout the year. This is the first time that an opportunity has occurred of obtaining continuous observations in one place for so long a time in these regions. The results of the work carried on in the lagoon of the Great Barrier Reef enables a comparison to be made between the conditions in the tropies and those in temperate waters. The water samples used were from various depths, forming part of the routine work at the plankton and hydrographic stations taken by the Nansen-Petterson water bottle. Outside samples were also taken with a glass sample bottle. The material was centrifuged and counted (100-200 c.c. of each sample). Most of it was examined when fresh, thus allowing the small naked dinoflagellates and coccolithophores to be included; the remainder was preserved in strong Fleming solution.

The organisms were grouped so far as possible in their genera. Neritic forms are predominant as was to be expected, especially among the diatoms, which were the most important group. Dinoflagellates and coccolithophores occurred in fair numbers, the latter restricted to water of high salinity. Pennate diatoms were unexpectedly numerous inshore, most of them being bottom forms stirred up from below.

The chief feature of the Great Barrier Reef plankton is that there is no special seasonal maximum and minimum ; it is maintained at much the same level throughout the year. This is strikingly different from the conditions in temperate waters where one or two maxima constantly occur, the phytoplankton maximum being followed by a zooplankton maximum. Miss Marshall finds no real seasonal change in the composition of the diatom flora. Large increases

*British Museum (Natural History). Great Barrier Reef Expedition 1928-29. Scientife Reports. Vol. 2. No. 4. (a) "Variation in some Chemical Conditions on and near Low Isles Reef", (b) "The Temperature of the Waters in the Anchorage, Low Isles". (c) "Physical and Chemical Conditions in the Mangrove Swamps". By A. P. Or the Great Barrier Reef Region". By Sheina M. Marshall. 1933. sometimes occur, but not regularly. In the lagoon the wind keeps the water thoroughly mixed from top to bottom. The nutrient salts estimated were present only in very small quantities throughout the year and no relation was found between them and the diatom or dinoflagellate abundance. The type of plankton production found, the numbers low and varying little during the year, depends largely on physical conditions:

Trichodesmium occurs in large patches at irregular intervals, being most abundant in the calmer months from October to February. On August 22-25 large patches drifted on to the reef causing great distress to the fishes in the moats, and eventually decayed along the shore. In Mr. Orr's report (No. 4a), it is stated that at this time, because of Trichodesmium, although the water had been supersaturated with oxygen just when the tide left the reef flat, it was completely denuded of oxygen before the tide re-entered. Only pools in which the blue-green alga was deposited in quantity and decomposing showed this absence of oxygen. On no other occasion was a value below saturation with respect to oxygen found during the day on the flat.

The changes in temperature, salinity, oxygen and $p H$ value among the coral reefs are specially described and vary enormously, both daily and according to season. These changes may have an important effect on the reef organisms. Tidal and diurnal changes are on the whole more important than seasonal changes. The greatest differences occurred in coral pools isolated at low tide either during the night or during the day, the water being usually supersaturated with oxygen during the day and undersaturated at night.

In the mangrove swamps (No. $4 c$ ) the diurnal and seasonal changes are greatest at spring tides and are greater in summer than in winter. There are diurnal fluctuations in temperature, salinity, $p \mathrm{H}$ value and oxygen content in the mangrove swamps on Low Isles reef flat and usually a night fall and a day rise in temperature : salinity rises at low tide whilst $p \mathbf{H}$ value and oxygen saturation fall. 